$\begin{array}{ll}\text { Research Square } & \begin{array}{l}\text { Preprints are preliminary reports that have not undergone peer review. } \\ \text { They should not be considered conclusive, used to inform clinical practice, } \\ \text { or referenced by the media as validated information. }\end{array}\end{array}$

\title{
Cumulative live birth rates between GnRH-agonist long and GnRH-antagonist protocol in one ART cycle when all embryos transferred: Real-Word Data of 18853 women from China
}

\author{
Jingwei Yang ( $\nabla$ teresa_lovelife@163.com ) \\ Chongqing Institute of Reproductive and Genetic: Chongqing Health Center for Women and Children \\ Xiaodong Zhang \\ Chongqing Institute of Reproductive and Genetic: Chongqing Health Center for Women and Children \\ Xiaoyan Ding \\ Chongqing Institute of Reproductive and Genetic: Chongqing Health Center for Women and Children

\section{Guoning Huang} \\ Chongqing Institute of Reproductive and Genetic: Chongqing Health Center for Women and Children \\ Hong Ye \\ Chongqing Institute of Reproductive and Genetic: Chongqing Health Center for Women and Children
}

\section{Research Article}

Keywords: gonadotropin releasing hormone agonist long protocol, gonadotropin releasing hormone antagonist protocol, cumulative live birth rate, ART cycle, embryo transfer

Posted Date: May 13th, 2021

DOI: https://doi.org/10.21203/rs.3.rs-501185/v1

License: (c) (1) This work is licensed under a Creative Commons Attribution 4.0 International License. Read Full License

Version of Record: A version of this preprint was published at Reproductive Biology and Endocrinology on August 12th, 2021. See the published version at https://doi.org/10.1186/s12958-021-00814-0. 


\section{Abstract}

Background: A consensus has been reached on the preferred primary outcome of all infertility treatment trials, which is the cumulative live birth rate (CLBR). Some recent randomized controlled trials (RCTs) and retrospective studies have compared the effectiveness of GnRH-antagonist and GnRH-agonist protocols but showed inconsistent results. Studies commonly used conservative estimates and optimal estimates to described the CLBR of one incomplete ART cycle and there are not many previous studies with data of the complete cycle to compare CLBRs in GnRH-antagonist versus $\mathrm{GnRH}$-agonist protocols.

Methods: A total of 18853 patients have completed their first IVF cycle including fresh and subsequent frozen-thawed cycles during 2016-2019, 16827 patients were treated with $\mathrm{GnRH}$-a long and 2026 patients with $\mathrm{GnRH}$-ant protocol. Multivariable logistic analysis was used to evaluate the difference of GnRH-a and GnRH-ant protocol in relation to CLBR.

Results: Before PSM, significant differences were observed in baseline characteristics and the CLBR was $50.91 \%$ in the $\mathrm{GnRH}$-a and $33.42 \%$ in the GnRH-ant (OR: $2.07 ; 95 \% \mathrm{Cl}: 1.88-2.28 ; P<0.001)$. Stratified analysis showed the CLBR of GnRH-ant was lower than $\mathrm{GnRH}$-a in suboptimal responders(46.89\% vs $27.42 \%$, $\mathrm{OR}=2.34,95 \% \mathrm{Cl}=1.99-2.74 ; P<0.001)$ and no differences of CLBR were observed in other patients between protocols. After adjusting for potential confounders, multivariable logistic analysis found the CLBR of GnRH-ant group was lower than that of $\mathrm{GnRH}$-a group (OR=2.11, 95\% Cl:1.69-2.63, $P<0.001)$. After $\mathrm{PSM}$ to balence the baseline between groups, the CLBR of $\mathrm{GnRH}$-a group was higher than that of $\mathrm{GnRH}$-ant group in suboptimal responders((38.61\% vs $28.22 \%$, $\mathrm{OR}=1.60,95 \% \mathrm{Cl}=1.28-1.99 ; P<0.001)$ and the normal fertilization rate and number of available embryo in $\mathrm{GnRH}$-a were higher than these of $\mathrm{GnRH}$-ant groups in suboptimal responders $(77.39 \%$ vs $75.22 \% ; 2.86 \pm 1.26$ vs $2.61 \pm 1.22 ; P<0.05)$. No significant difference was observed in other patients between different protocols.

Conclusions: It is crucial to optimize the utilization of protocols in different ovarian response patients and reconsider the field of application of GnRH-ant protocols in China.

\section{Background}

With the development of gonadotropin-releasing hormone agonist ( $\mathrm{GnRH}-\mathrm{a}$ ) in the 1980s (1), its role in controlled ovarian stimulation (COS) has been remarkable. The standard GnRH-a long protocol is the vital one in China due to its association with steady and higher clinical pregnancy rates in fresh embryo transfer (ET) in patients undergoing in vitro fertilization (IVF) (2). Recently, GnRH antagonist (GnRH-ant) protocol is widely adopted because of its short treatment duration, lower gonadotropin requirement and reduction in the incidence of severe ovarian hyperstimulation syndrome (OHSS) rate than GnRHa protocol (3).

Some recent randomized controlled trials (RCTs) and retrospective studies have compared the effectiveness of both protocols but showed inconsistent results (4-7). So, it is necessary to focus on finding important indicator for making decisions and should be considered as a key point in defining the success of assisted reproductive technology (ART) treatment. This not only reflects the outcome of an embryo transfer, such as pregnancy rate, abortion rate, but also evaluates the potency of all embryos after one oocyte retrieval cycle.

A consensus has been reached on the preferred primary outcome of all infertility treatment trials, which is the live birth rate or cumulative live birth rate (CLBR). It is defined as the live birth per women for over a defined time period (or number of treatment cycles) in 2013 (8). CLBR requires a long duration period including fresh and subsequent frozen-thawed cycles from the initial oocyte retrieval and there are varying equations to calculate CLBR (9). Moreover, it might help patients to make decisions for continuing treatment or remaining childless.

Nowadays, the unique medical characteristics of the patients are stored in electronic medical records (EMRs). However, the data from EMRs has less regulatory acceptance as compared to those from RCTs due to weaknesses such as missing information and observational bias, which in turn facilitates the quantity, accessibility, and heterogeneity of offline observational data for a variety of diseases and patients (10).

Hence, this study aimed to compare the CLBR of different number of retrieved oocytes between GnRH-a and GnRH-ant protocol after one complete ART cycle with all embryos used. This approach is advantageous and can be recommended to doctors in choosing an appropriate protocol for patients with more accurate probability of live births.

\section{Methods}

This is a retrospective real-world data study, which links the information of patients from the EMRs, Chongqing Health Center for Women and Children database to evaluate women who commenced their first COS cycle [including IVF and Intracytoplasmic Sperm Injection (ICSI)] including fresh and subsequent frozen-thawed cycles with no embryos left from January 2016 to December 2019. All patients included in this study were undergoing their first COS cycle with $\mathrm{GnRH}$-a protocol or $\mathrm{GnRH}$-ant protocol. Patients with preimplantation genetic testing (PGT) cycles, chromosome abnormalities and uterine malformation were excluded. Finally, a total of 18853 patients were analyzed, and 16827 patients underwent treatment with GnRH-a long protocol and 2026 patients underwent treatment with GnRH-ant protocol (Fig. 1).

The study involving human participants was reviewed and approved by the Ethics Committee Review Board of Chongqing Health Center for Women and Children (approval number: 2020-RGI-13) for retrospective analysis and clinical data reporting. Informed consent was waived by the committee because of the retrospective nature of the study.

\section{GnRH-agonist long protocol}


GnRH-a (Triptorelin $0.1 \mathrm{mg} / \mathrm{d}$ or $0.05 \mathrm{mg} / \mathrm{d}$, sc. Decapeptyl Ferring, Germany) was used for pituitary downregulation from the previous luteal phase. After administration of $\mathrm{GnRH}$ agonist for $14-21$ days, if the levels of estrogen $<50 \mathrm{pg} / \mathrm{mL}$, luteinizing hormone $<5 \mathrm{mlU} / \mathrm{mL}$ and $\mathrm{P}<1 \mathrm{ng} / \mathrm{mL}$, then a dose of recombinant follicle stimulating hormone (rFSH) ranging from 75 to $300 \mathrm{IU}$ was administrated subcutaneously per day based on the woman's age, antimüllerian hormone (AMH) level and antral follicle counts (AFC).

\section{GnRH-antagonist protocol}

COS was initiated on day 2 or 3 of the cycle with a dose of rFSH ranging from 75 to $300 \mathrm{IU}$. GnRH antagonist of $0.25 \mathrm{mg}$ (Orgalutran, Organon, The Netherlands or Cetrorelix, Merck serono, Switzerland) was given to patients daily if at least one of the following criteria was fulfilled: (i) with at least one follicle of $>14 \mathrm{~mm}$; (ii) serum estrogen level of $>600 \mathrm{pg} / \mathrm{mL}$; and (iii) serum LH level of $>10 \mathrm{IU} / \mathrm{L}$ (11).

\section{Trigger day}

If there were at least three follicles were measured $>18 \mathrm{~mm}$ in diameter, human chorionic gonadotropin (hCG, Merck Serono, Italy) was administered and part of $\mathrm{GnRH}$-ant cycles used Triptorelin Acetate Injection (GnRH-a, Ferring GmbH, Germany) as trigger. After that, transvaginal oocyte retrieval was performed 36h, and then embryo transfer (ET) was performed on day 3 after oocyte retrieval. Luteal-phase support was started immediately after oocyte retrieval with vaginal combined oral progesterone. Most of the patients received double embryo transfer (DET) unless the patient had only one available embryo for single embryo transfer according to the national guidelines (12). Surplus available embryos or all-frozen embryos (due to OHSS, thin endometrium, abnormal blood biochemical index and personal causes of patients) were frozen for later transfer in subsequent frozen embryo transfer (FET) cycles. The vast majority of these embryos were frozen on day 3. Embryos that are not suitable for cryopreservation on day 3 were cultured till days 5 or 6 and vitrified if they reach the blastocyst stage. Luteal-phase support with vaginal combined oral progesterone was started three days before FET.

\section{Vitrification and storage}

The cryotop vitrification method was performed according to the manufacturer's instructions as reported by Kuwayama et al (13). The Kitazato vitrification kit (Kita, Toyota, Japan) was used for vitrification. Embryos were equilibrated in VS1 solution for 5 min before exposure to vitrification solution (VS 2). The embryos were dipped in vitrification solution (VS 2) for $30 \mathrm{~s}$, and the vitrification procedure was carried out according to the manufacturer's protocol. The embryos were then placed on a Cryotop sheet and excess vitrification solution was removed by aspiration using a pipette before the immersion of the carrier in LN2.

\section{Warming procedure}

A Dewar of LN2 containing the carriers was placed close to the microscope. Forceps were used to grasp the straw in the LN2 and placed it in a dish containing $3 \mathrm{~mL}$ of $1.0 \mathrm{~mol} / \mathrm{L}$ sucrose at $37^{\circ} \mathrm{C}$ for $1 \mathrm{~min}$. All the embryos were then transferred sequentially to 0.5 and $0.25 \mathrm{~mol} / \mathrm{L}$ sucrose solutions at room temperature for 3 min each. The embryos were then washed several times in Quinn's 1024 (Cooper Surgical, CT, USA) solution, and placed in G1 medium (Vitrolife, Kungsbacka, Sweden) for further culturing. Post-warming survival of cryopreserved embryos was defined as survival of more than one-half of the original cells that are intact.

If pregnancy is achieved, then luteal phase support was continued until 12 weeks' gestation in both the groups.

\section{Outcome measures}

The indexes of embryo quality were the D2-4c (Day 2-4 cell) rate and D3-8c (Day 3-8 cell) rate defined as the proportion of embryos with 4/8 cells on Day 2/3 of the total number of two pronuclei (2PN) cleavege embryos. The primary outcome was the cumulative live birth per Ovum Pick-Up (OPU), which was defined as the first live born baby at $\geq 28$ weeks' gestation that results from a completed ART cycle, including all fresh and FETs that result from the associated ovarian stimulation. If a live birth occurs, then the patients can obtain an outcome regardless of subsequent cycles. According to this definition, multiple deliveries from the same pregnancy or multiple live births were considered as one live birth. CLBRs were calculated as the proportion of cycles that achieved the first live birth.

\section{Statistics}

Data are presented as means (SD) or number (\%) as appropriate. Wilcoxon rank-sum test was used for analyzing continuous variables. The cumulative pregnancy rates and CLBRs in the $\mathrm{GnRH}$-a and $\mathrm{GnRH}$-ant groups were compared by Chi-square test. The number of oocytes retrieved were categorized into five groups, namely 1-3 (poor), 4-9 (suboptimal), 10-15 (normal) and >16 (high) [14]. The Cochran-Mantel-Haenszel (CMH) test by different levels of retrieved oocytes was used to compare the CLBR, normal fertilization rate, D2-4c rate and D3-8c rate between protocol groups. A multivariable logistic regression analysis were used to evaluate the relative prognostic significance of protocol groups, female age, body mass index (BMI), AMH, FSH, the number of retrieved oocytes, the number of available embryos, type of infertility in relation to CLBR. Interactions between independent covariates were adjusted. Utilized Propensity Score Matching (PSM) for sampling by up to 1:1 nearest neighbor matching with caliper (0.05) to balence the baseline between groups. $P$-values of $<0.05$ were considered to indicate statistical significance. All analyses were conducted using Stata (version 15.1, College Station, TX: StataCorp LLC).

\section{Results}

Following planned exclusions, 18853 women were included in this analysis (Fig. 1). Epidemiological, clinical, and biological characteristics of the population before and after PSM are summarized in Table 1. Before PSM, significant differences were observed between the two protocols in age, infertility duration, BMI, $\mathrm{AMH}, \mathrm{FSH}, \mathrm{Gn}$ dose, Gn day, retrieved oocytes, available embryos, type of infertility. After 1:1 nearest neighbor PSM matching, the analysis focused on 3439 
patients (1704 patients in GnRH-a group and 1735 patients in $\mathrm{GnRH}$-ant group) and the mean age of the study population was $34.06 \pm 4.94$ years. No differences in age, infertility duration, $\mathrm{BMI}, \mathrm{AMH}, \mathrm{FSH}, \mathrm{Gn}$ dose, available embryos, cause of infertility between groups. Significant differences between the two comparative groups were observed in $\mathrm{Gn}$ days (10.14 \pm 1.61 in $\mathrm{GnRH}$-a vs $9.89 \pm 1.58$ in $\mathrm{GnRH}$-ant groups; $P<0.001)$, number of oocytes retrieved(10.43 \pm 6.28 in $\mathrm{GnRH}$-a vs $9.68 \pm 7.11$ in $\mathrm{GnRH}$-ant groups; $P<0.001)$ and available embryo(3.92 \pm 2.69 in $\mathrm{GnRH}$-a vs $3.68 \pm 2.76$ in $\mathrm{GnRH}$-ant groups; $P<0.001)$. Patient assignment before and after PSM is reported in Supplemental Fig. 1.

Table 1

Baseline characteristics of women at first IVF cycle treated with either GnRH-agonist or GnRH-antagonist protocol and after PSM.

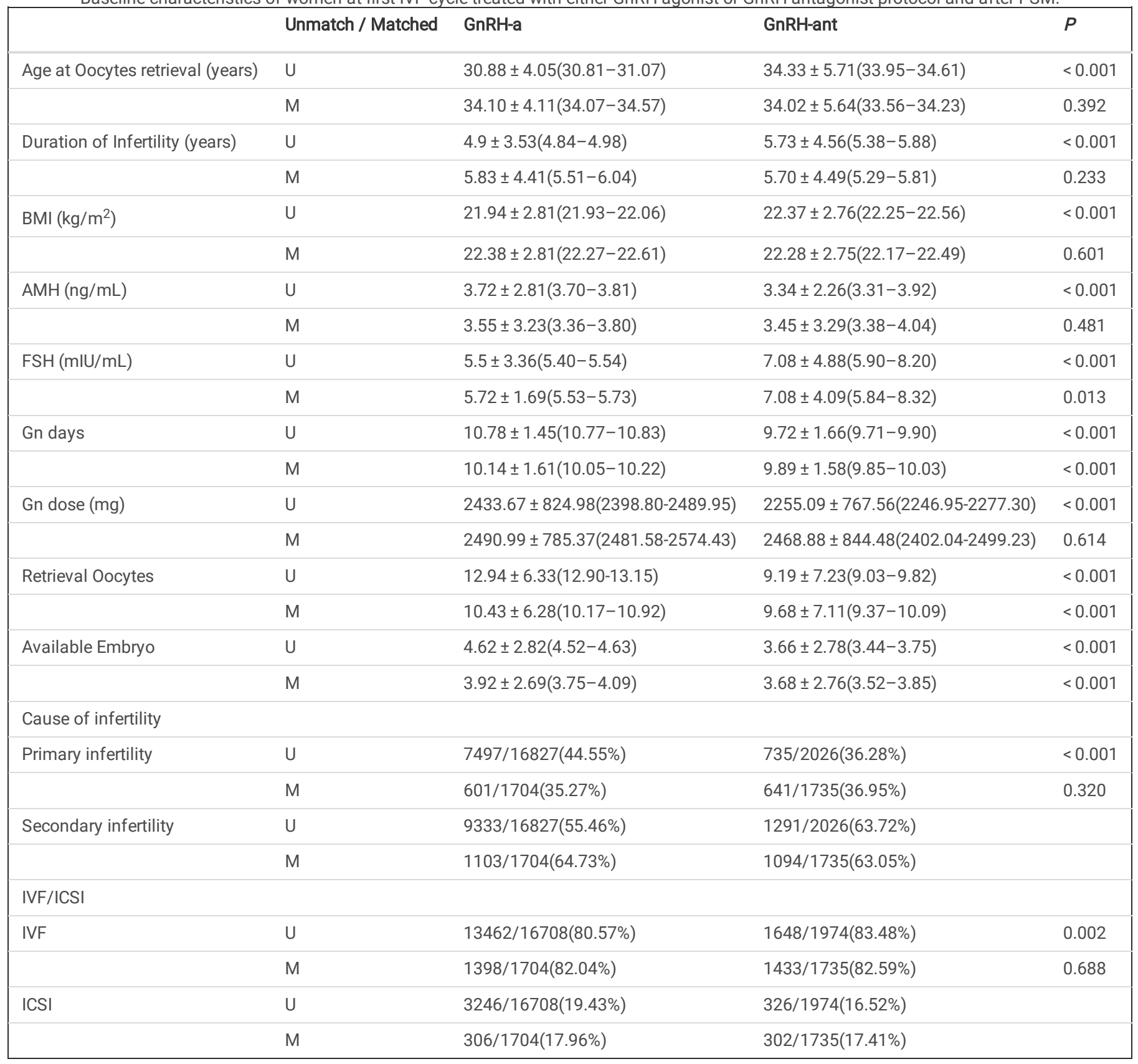

The CLBR after one complete ART cycle was 8567/16827 (50.91\%) in the $\mathrm{GnRH}$-a group and 677/2026 (33.42\%) in the GnRH-ant group (OR = 2.07; $95 \%$ Cl: 1.88-2.28; $P<0.001)$. Stratified analysis of CLBR was performed after grouping number of oocytes retrieved. There were higher $\mathrm{CLBR}$ in $\mathrm{GnRH}-\mathrm{a}$ than $\mathrm{GnRH}-$ ant in poor responders $(25.72 \%$ vs $18.13 \%, \mathrm{OR}=1.56,95 \% \mathrm{Cl}=1.13-2.16, P=0.007)$ and suboptimal responders $(46.89 \% \mathrm{vs} 27.42 \%, \mathrm{OR}=2.34,95 \% \mathrm{Cl}=1.99-$ $2.74 ; P<0.001)$. After $1: 1$ nearest neighbor PSM matching, the CLBR was $689 / 1704(40.43 \%)$ in the GnRH-a group and $621 / 1735(35.79 \%)$ in the $\mathrm{GnRH}$-ant group $(\mathrm{OR}=1.22 ; 95 \% \mathrm{Cl}: 1.06-1.40 ; P=0.006)$. In $\mathrm{CMH}$ stratified analysis, there were significant differences in CLBR which of $\mathrm{GnRH}$-a group was higher than that of $\mathrm{GnRH}$-ant group in suboptimal responders(38.61\% vs $28.22 \%, \mathrm{OR}=1.60,95 \% \mathrm{Cl}=1.28-1.99 ; P<0.001)$. No differences in $\mathrm{CLBR}$ between protocols in other subgroup(Table 2). 
Table 2

Stratified analysis of CLBR after grouping age and number of retrieval oocytes

\begin{tabular}{|lllll|}
\hline Age & Before PSM & \multicolumn{3}{l|}{ After PSM } \\
\hline Ovarian Response & GnRH-a & GnRH-ant & GnRH-a & GnRH-ant \\
\hline No embryo to transfer & 362 & 70 & 87 & 36 \\
\hline Poor & $151 / 587(25.72 \%)^{*}$ & $68 / 375(18.13 \%)^{*}$ & $29 / 150(19.33 \%)$ & $60 / 314(19.11 \%)$ \\
\hline Suboptimal & $2194 / 4679(46.89 \%)$ & $241 / 879(27.42 \%)^{*}$ & $266 / 689(38.61 \%) *$ & $217 / 769(28.22 \%)^{*}$ \\
\hline Normal & $3370 / 6105(55.20 \%)$ & $185 / 356(51.97 \%)$ & $232 / 467(49.68 \%)$ & $176 / 317(55.52 \%)$ \\
\hline High & $2852 / 5094(55.99 \%)$ & $183 / 346(52.89 \%)$ & $162 / 311(52.09 \%)$ & $168 / 299(56.19 \%)$ \\
\hline * Before PSM, poor responders: GnRH-a:GnRH-ant, OR=1.56, 95\%Cl=1.13-2.16, $P=0.007 ;$ & \\
\hline * Before PSM, suboptimal responders: GnRH-a:GnRH-ant, OR=2.34, 95\%Cl=1.99-2.74, $P<0.001 ;$ \\
\hline *After PSM, suboptimal responders: GnRH-a:GnRH-ant, OR=1.60, 95\%Cl=1.28-1.99, $P<0.001 ;$ \\
\hline
\end{tabular}

Considering the results of stratified analysis, multivariable logistic analysis based on the four subgroups of ovarian response (poor; suboptimal; normal; high) was performed. After adjusting for potential confounders (such as age, infertility duration, BMI, AMH, FSH, available embryos, cause of infertility, IVF/ICSI) in different ovarian responders, the protocol acts as an independent influential factor of CLBR for suboptimal ovarian responders. Significant difference was found the CLBR of GnRH-ant group was lower than that of $\mathrm{GnRH}$-a group (GnRH-a:GnRH-ant, OR $=2.11,95 \% \mathrm{Cl}: 1.69-2.63 ; P<0.001)$. In addition, age and available embryo were the important factors for each subgroups (Table 3). After PSM matching, adjusting for potential confounders (age, FSH, available embryo, retrieval oocyte and Gn day) in different ovarian responders, the protocol acts as an independent influential factor of CLBR for suboptimal ovarian responders(GnRH-a:GnRH-ant, OR=1.84, 95\%Cl:1.37-2.47; $P<0.001)$ (Supplemental Table 1).

Table 3

Multivariable logistic regression of CLBR in different oocyte retrieval groups after adjusting for potential confounders(Before PSM)

\begin{tabular}{|c|c|c|c|c|}
\hline & Poor & Suboptimal & Normal & High \\
\hline Group (1 = GnRH-agonist; 2 = GnRH-antagonist) & $1.15(0.70-1.88)$ & $2.11(1.69-2.63)^{\star}$ & $1.04(0.78-1.38)$ & $1.01(0.98-1.03)$ \\
\hline Age & $0.89(0.84-0.94)^{\star}$ & $0.93(0.91-0.94)^{\star}$ & $0.93(0.92-0.95)^{\star}$ & $0.96(0.94-0.98)^{*}$ \\
\hline $\mathrm{AMH}$ & $0.91(0.78-1.07)$ & $1.00(0.97-1.04)$ & $1.02(0.99-1.04)$ & $1.06(1.04-1.09)^{\star}$ \\
\hline BMI & $0.97(0.90-1.04)$ & $0.98(0.95-1.01)$ & $0.96(0.94-0.99)^{\star}$ & $0.99(0.97-1.02)$ \\
\hline Available Embryo & $1.47(1.02-2.12)^{\star}$ & $1.32(1.25-1.39)^{\star}$ & $1.19(1.14-1.23)^{\star}$ & $1.10(1.07-1.13)^{\star}$ \\
\hline IVF/ICSI & $0.93(0.46-1.85)$ & $1.08(0.88-1.33)$ & $1.20(1.01-1.44)^{\star}$ & $1.39(1.14-1.66)^{*}$ \\
\hline \multicolumn{5}{|c|}{$\begin{array}{l}\text { Results showing the parameters with significant differences after adjusting for potential confounders (such as age, infertility duration, BMI, AMH, FSH, } \\
\text { available embryo, cause of infertility and IVF/ICSI). }\end{array}$} \\
\hline
\end{tabular}

After 1:1 nearest neighbor PSM matching, by stratified retrieval oocyte, the analysis of embryo quality showed that the normal fertilization rate and number of available embryo in $\mathrm{GnRH}$-a were higher than these of $\mathrm{GnRH}$-ant groups in suboptimal responders (77.39\% vs $75.22 \% ; 2.61 \pm 1.22$ vs $2.61 \pm 1.22 ; P<0.05)$. No significant difference was observed in normal fertilization rate and D2-4c rate, D3-8c rate between different protocols (Table 4). 
Table 4

Embryo quality of patients treated with GnRH-a or GnRH-ant protocol(After PSM).

\begin{tabular}{|c|c|c|c|c|c|c|c|c|}
\hline \multirow[t]{2}{*}{$\begin{array}{l}\text { Ovarian } \\
\text { Response }\end{array}$} & \multicolumn{2}{|c|}{$\begin{array}{l}\text { Available } \\
\text { embryo }\end{array}$} & \multicolumn{2}{|c|}{ Normal fertilization rate } & \multicolumn{2}{|l|}{ D2-4c rate } & \multicolumn{2}{|l|}{ D3-8c rate } \\
\hline & $\begin{array}{l}\text { GnRH- } \\
\text { a }\end{array}$ & $\begin{array}{l}\text { GnRH- } \\
\text { ant }\end{array}$ & $\mathrm{GnRH}-\mathrm{a}$ & GnRH-ant & $\mathrm{GnRH}-\mathrm{a}$ & GnRH-ant & GnRH-a & GnRH-ant \\
\hline Poor & $\begin{array}{l}1.55 \\
\pm 0.56\end{array}$ & $\begin{array}{l}1.56 \\
\pm 0.58\end{array}$ & 221/294(75.17) & $472 / 639(73.86)$ & 127/219(57.99) & 274/457(59.96) & $89 / 219(40.64)$ & $162 / 457(3$ \\
\hline Suboptimal & $\begin{array}{l}2.86 \\
\pm \\
1.26^{\mathrm{a}}\end{array}$ & $\begin{array}{l}2.61 \\
\pm \\
1.22^{\mathrm{b}}\end{array}$ & $3115 / 4025(77.39)^{*}$ & $3076 / 4089(75.22)^{*}$ & $1847 / 3034(60.88)$ & $1814 / 2984(60.79)$ & $1200 / 3034(39.55)$ & $1109 / 298$ \\
\hline Normal & $\begin{array}{l}4.41 \\
\pm 2.01\end{array}$ & $\begin{array}{l}4.50 \\
\pm 2.20\end{array}$ & $3894 / 5036(77.32)$ & 2635/3430(76.82) & 2250/3777(59.57) & $1559 / 2554(61.04)$ & $1421 / 3777(37.62)$ & $987 / 25541$ \\
\hline High & $\begin{array}{l}7.08 \\
\pm 3.36\end{array}$ & $\begin{array}{l}7.11 \\
\pm 3.39\end{array}$ & 4318/5658(76.32) & $4315 / 5630(76.64)$ & 2595/4228(61.37) & $2498 / 4211(59.32)$ & $1430 / 4228(33.82)$ & $1377 / 420^{\circ}$ \\
\hline
\end{tabular}

a vs b: $P<0.001$;

*:GnRH-a:GnRH-ant, OR(95\%Cl) = 1.127(1.018-1.249); $P=0.023$;

\section{Discussion}

$\mathrm{GnRH}$-ant protocol is widely used because of its short treatment duration, and lower gonadotropin requirement (3). This protocol offers an available alternative to the long agonists, providing a shorter duration of treatment with fewer injections and with no adverse effects on ART outcome (15). Our study showed that $\mathrm{GnRH}$-ant protocol involves less Gn days [(9.72 \pm 1.66$) \mathrm{d}$ vs $(10.78 \pm 1.45) \mathrm{d}]$ as well as Gn dose [(2255.09 \pm 767.56$) \mathrm{IU}$ vs $(2433.67 \pm 824.98) \mathrm{IU}]$ than $\mathrm{GnRH}$-a protocol. In addition, OHSS is a preventable condition and implementation of evidence-based prevention strategies enables clinicians to reduce its occurrence, and there are many evidences showing significantly lower rate of OHSS in GnRH-ant protocol (16-18).

Considering these outcomes, it is still controversial on the efficacy of GnRH-a and GnRH-ant protocols. The main finding of this study was that the CLBR was comparable in both $\mathrm{GnRH}$-a and $\mathrm{GnRH}$-ant groups, with $50.91 \%$ and $33.42 \%$ achieving the first live birth. After adjusting for potential confounders, the CLBR of $\mathrm{GnRH}$-ant group was lower than that of $\mathrm{GnRH}$-a group in suboptimal ovarian responders. But for other patients, no difference in CLBR was found between the two protocols. The CLBR presented in this study was based on previous studies, which showed lower pregnancy rate, ongoing pregnancy rate and LBR of fresh transfer cycles in the $\mathrm{GnRH}$-ant protocol than in the $\mathrm{GnRH}$-a protocol $(2,16)$. While other studies showed no significant difference in clinical pregnancy rates, ongoing pregnancy rate and LBR between $\mathrm{GnRH}$-a protocol and $\mathrm{GnRH}$-ant protocol $(3,19,20)$.

A RCT by M.Toftager et al (7) showed no difference in baseline characteristics, and revealed CLBR to be $34.1 \%$ in the GnRH-ant group versus $31.2 \%$ in the $\mathrm{GnRH}$-a group (OR:1.14; $95 \% \mathrm{Cl}: 0.88-1.48, P=0.32)$. Though there was a difference with our study results, the presence of population differences is a negligible issue. Indeed, pivotal RCTs often lacked external validity as eligibility criteria excluded some of the patient subgroups who were commonly treated in real-world clinical practice (21). In China, by considering the financial burden of patients, and in order to improve the success rate of each ET, the GnRH-a protocol is still regarded as the main protocol but the comfort of treatment was not taken into account. The GnRH-ant protocol was mostly recommended for patients who are more prone to OHSS. The population differences demonstrated an effect on objective comparison of two different treatment conditions. The impact of age and oocytes retrieval on CLBR has been confirmed in several prospective studies published in recently (22-24). Other studies have found that the retrieved oocytes showed positive association with the number of euploid embryos that are available for transfer. These findings provide an explanation with regard to higher availability of euploid embryos for transfer, resulting in increased CLBR from higher oocyte yields $(25,26)$.

Selecting an ovarian stimulation protocol for patients with poor ovarian response is difficult because of the retrieval of fewer oocytes. M-C Huang et al (5) have observed that the $\mathrm{GnRH}$-a protocol showed correlation with higher LBR and implantation rate than the GnRH-ant protocol for ET cycles in patients with diminished ovarian reserve (DOR), suggesting that only a few oocytes can be retrieved from these patients in a single stimulation cycle. The LBR and implantation rate showed association with endometrial and embryo quality. In our results, due to the differences of normal fertilization rate and available embryo but no difference of embryo quality, unsynchronization of follicle development (27) and decreased endometrial receptivity of GnRH-ant protocol might be related to the clinical outcomes $(28,29)$.

There is asynchrony in antral follicle development during early follicular phase (30) which is further expanded in progress of COS. Seifer DB have found that the proliferation of granulosa cells was obviously faster in follicle treated with $\mathrm{GnRH}$-a than blank control group (31). In the ovary, AMH is produced by the granulosa cells of early developing follicles and seems to be able to inhibit the initiation of primordial and FSH-induced follicle growth. Wang B et al speculated that in addition to the flare up effect of $\mathrm{GnRH}-\mathrm{a}$, $\mathrm{GnRH}-\mathrm{a}$ could reduce the AMH expression in small antral folliclea which increase the responsiveness to FSH and promote growth, however, the effect on larger follicles is not obvious (27, 32). Mei's research provided the new evidence that GnRHa and $\mathrm{GnRH}$-ant showed different effect on ovarian reserve and suggest that this discrepancy might be caused by different regulation on intraovairan autocrine/paracrine factors AMH and SCF through GnRHR-I system (33). Hernandez ER et al have shown that GnRH-ant might disrupt an auto/paracrine loop that is essential for mitotic program of the endometrial cells and it is manifested by a decrease in the pregnancy rates and increase in the abortion rates (34). Rackow BW et al have found HOXA10 (an essential regulator of endometrial receptivity) expression was significantly decreased in endometrial stromal cells in 
$\mathrm{GnRH}$-ant treated cycles when compared with $\mathrm{GnRH}$-a treated cycles or natural cycle controls (29). Ruan $\mathrm{HC}$ et a/ have found that GnRH-a, but not GnRH antagonist, have partially restored the endometrial physiological secretion and improved uterine receptivity in mice (35). A comparative proteomic analysis demonstrated that endometrial receptivity is more strongly impaired by $\mathrm{GnRH}$-ant than $\mathrm{GnRH}$-a protocols (36). The results of the above studies indicated that the endometrial receptivity of $\mathrm{GnRH}$-a protocol might be better than $\mathrm{GnRH}$-ant protocol.

For normal and high ovarian responder patients, GnRH-ant protocol group had lower levels of Gn dosage, days of Gn administered, number of retrieved oocytes, number of matured oocytes, normal fertilization rate (37) and substantial reduction in OHSS without reducing the likelihood of achieving live birth or ongoing pregnancy(19). In our study, all subgroup analyses for covariate's effect on the CLBR were based on post hoc analyses with multiple strata. Female age, IVF/ICSI and available embryo were the influential factors of CLBR in normal and high ovarian responder patients and the protocols showed no effect on the CLBR. From one OPU involves multiple transfer cycles and a very few patient received single embryo transfer, we found it had no effect on the CLBR. Wen Ding et al (38) have confirmed that female obesity has adversely affected the CLBR after utilizing the available embryos from first oocyte retrieval. But we found BMI as a unique influential factor in only normal ovarian responder patients, and so it should be interpreted with caution and findings should be further investigated.

However, this study has limitation due to its retrospective nature. Due to analysis of real-world data, the characteristics of patients were not balanced. Although the confounders were adjusted using mutivariable logistic regression and PSM for sampling to balence the baseline between groups, some potential confounders might be neglected. A well-designed, multicenter, prospective RCT is still warranted to further support our results. Different molecular mechanisms of endometrial receptivity between $\mathrm{GnRH}-\mathrm{a}$ and $\mathrm{GnRH}$-ant protocols should be evaluated for further study.

After one complete cycle, despite significantly higher CLBR in the GnRH-a protocol for suboptimal ovarian response patients, no significant difference of CLBR was observed between these two protocols in other patients. However, it is crucial to optimize the utilization of protocols in different ovarian response patients, personalized protocols for patients and reconsider the field of application of $\mathrm{GnRH}$-ant protocol in China.

\section{Conclution}

To provide evidence for protocol choosing, under a large sample study, our results suggest that despite significantly higher CLBR in the GnRH-a protocol for suboptimal ovarian response patients, no significant difference of CLBR was observed between these two protocols in other patients. It is crucial to optimize the utilization of protocols in different ovarian response patients, personalized protocols for patients and reconsider the field of application of GnRH-ant protocol in China.

\section{Abbreviations}

ART, assisted reproductive technology

AMH,anti-Müllerian hormone

AFC,antral follicle count

BMI, body mass index

COS, controlled ovarian stimulation

CLBR,cumulative live birth rate

ET,embryo transfer

EMRs, electronic medical records

FET,frozen-embryo transfer

GnRH-a, gonadotropin-releasing hormone agonist

GnRH-ant, gonadotropin-releasing hormone antagonist

IVF, in vitro fertilization

ICSI,intracytoplasmic sperm injection

OHSS,ovarian hyperstimulation syndrome

OPU,ovum-pick up

OCP, oral contraceptive pill

POR, poor ovarian response

PGT, preimplantation genetic testing 
PSM, Propensity Score Matching

RCTs, randomized controlled trials

rFSH,recombinant follicle-stimulating hormone

\section{Declarations}

\section{Ethical Approval and Consent to participate}

Research approval was granted by the ethics committee of Chongqing Health Center for Women and Children (ID:2020-RGI-13).

\section{Consent for publication}

Not applicable.

\section{Availability of supporting data}

The datasets used and/or analyzed during the current study are available from the corresponding author on reasonable request.

\section{Competing interests}

The authors declare that they have no conflict of interest.

\section{Funding}

There was no funding for this study.

\section{Authors' contributions}

The present work was designed by HY. Data extraction and analysis were performed by JY and XZ. JY and JY participated in the data collection. GH and HY participated in revisions to the article. All authors have read and approved the final manuscript.

\section{Acknowledgements}

We thank all the embryologists and nurses of Chongqing Reproductive and Genetics Institute for embryo culture, data recording, and other clinical assistance.

\section{References}

1. Fleming R, et al. A new systematic treatment for infertile women with abnormal hormone profiles. Br J Obstet Gynaecol. 1982; 37(7):481-482

2. Lambalk CB, et al. GnRH antagonist versus long agonist protocols in IVF: a systematic review and meta-analysis accounting for patient type. Hum Reprod Update. 2017, 23(5):560-579.

3. Wang R, et al. Comparisons of $\mathrm{GnRH}$ antagonist protocol versus $\mathrm{GnRH}$ agonist long protocol in patients with normal ovarian reserve: A systematic review and meta-analysis. PLoS One. 2017; 12(4):e0175985

4. Yang J. Clinical efficacy of GnRH antagonist protocol vs. GnRH-agonist down-regulation protocol in normal response patients and the relevant factors of occytes retrieved. Center for Clinical Reproductive Medicine. 2018

5. Huang MC, et al. GnRH agonist long protocol versus $\mathrm{GnRH}$ antagonist protocol for various aged patients with diminished ovarian reserve: A retrospective study. PLoS One. 2018; 13(11):e0207081.

6. Ruggiero M, et al. Comparison between $\mathrm{GnRH}$ agonist and antagonist protocols for severe endometriosis in assisted reproductive cycles. Journal of Endometriosis. 2012; 4(1):42-47.

7. Toftager $\mathrm{M}$, et al. Cumulative live birth rates after one ART cycle including all subsequent frozen-thaw cycles in 1050 women: secondary outcome of an RCT comparing GnRH-antagonist and GnRH-agonist protocols. Hum Reprod. 2017; 32(3):556-567.

8. Harbin Consensus Conference Workshop G, Conference C, Legro RS, et al. Improving the reporting of clinical trials of infertility treatments (IMPRINT): modifying the CONSORT statementdaggerdouble dagger. Hum Reprod. 2014; 29:2075-2082.

9. Maheshwari A, et al. Cumulative live birth rate: time for a consensus? Hum Reprod. 2015; 30(12):2703-2707.

10. Bica I, et al. From real-world patient data to individualized treatment effects using machine learning: Current and future methods to address underlying challenges. Clinical Pharmacology \& Therapeutics. 2020; 109(1):87-100

11. Lainas T, et al. In a flexible antagonist protocol, earlier, criteria-based initiation of GnRH antagonist is associated with increased pregnancy rates in IVF. Hum Reprod. 2005; 20(9):2426-2433.

12. Sun $Y \mathrm{~J}$, et al. Chinese expert consensus on numbers of embryos transferred.

13. Chian RC, et al. High survival rate of bovine oocytes matured in vitro following vitrification. J Reprod Dev. 2004; 50(6):685-696.

14. Society for Assisted Reproductive Technology, American Society for Reproductive Medicine. Assisted reproductive technology in the United States: 2000 results generated from the American Society for Reproductive Medicine/Society for Assisted Reproductive Technology Registry. Fertil Steril. 2004; 
81(5):1207-1220.

15. Copperman AB, et al. Optimal usage of the GnRH antagonists: a review of the literature. Reprod Biol Endocrinol. 2013; $11(1): 20$.

16. Al-Inany HG, et al. Gonadotrophin-releasing hormone antagonists for assisted conception: a Cochrane review. Reprod Biomed Online. 2007; 14(5):640649.

17. Al-Inany HG, et al. Gonadotrophin-releasing hormone antagonists for assisted reproductive technology. Cochrane Database Syst Rev. 2016; 4:CD001750.

18. Xiao J, et al. Effectiveness of GnRH antagonist in the treatment of patients with polycystic ovary syndrome undergoing IVF: a systematic review and meta analysis. Gynecol Endocrinol. 2013; 29(3):187-191.

19. Al-Inany HG, et al. Gonadotrophin-releasing hormone antagonists for assisted reproductive technology. Cochrane Database Syst Rev. 2011 ;(5): CD001750.

20. Kolibianakis EM, et al. Among patients treated for IVF with gonadotrophins and GnRH analogues, is the probability of live birth dependent on the type of analogue used? A systematic review and meta-analysis. Hum Reprod Update. 2006; 12(6):651-671.

21. Pasello G, et al. Real world data in the era of Immune Checkpoint Inhibitors (ICls): Increasing evidence and future applications in lung cancer. Cancer Treat Rev. 2020; 87:102031.

22. Racca A, et al. Do we need to measure progesterone in oocyte donation cycles? A retrospective analysis evaluating cumulative live birth rates and embryo quality. Hum Reprod. 2020; 35(1):167-174.

23. Law YJ, et al. The number of oocytes associated with maximum cumulative live birth rates per aspiration depends on female age: a population study of 221221 treatment cycles. Hum Reprod. 2019; 34(9):1778-1787.

24. Polyzos NP, et al. Cumulative live birth rates according to the number of oocytes retrieved after the first ovarian stimulation for in vitro fertilization/intracytoplasmic sperm injection: a multicenter multinational analysis including approximately 15,000 women. Fertil Steril. 2018; 110(4):661670 e661.

25. Labarta E, et al. A Higher Ovarian Response after Stimulation for IVF Is Related to a Higher Number of Euploid Embryos. Biomed Res Int. 2017 ; 5637923.

26. Venetis CA, et al. Is more better? A higher oocyte yield is independently associated with more day-3 euploid embryos after ICSI. Hum Reprod. 2019; 34(1):79-83.

27. Wang B, et al. Appropriate prolongation of GnRH-a down-regulation improves the synchronism of follicular development. Zhonghua Nan Ke Xue. 2011; 17(12):1087-1091.

28. Bahceci M, et al. Frozen-thawed cleavage-stage embryo transfer cycles after previous GnRH agonist or antagonist stimulation. Reprod Biomed Online. $2009 ; 18(1): 67-72$.

29. Rackow BW, et al. GnRH antagonists may affect endometrial receptivity. Fertil Steril. 2008; 89(5):1234-1239.

30. Gougeon A, et al. Evolution of the diameters of the largest healthy and atretic follicles during the human menstrual cycle. J Reprod Fertil. 1983; 69(2):497502.

31. Seifer DB, et al. Gonadotropin-releasing hormone agonist-induced differences in granulosa cell cycle kinetics are associated with alterations in follicular fluid mullerian-inhibiting substance and androgen content. J Clin Endocrinol Metab. 1993; 76(3):711-714.

32. Huang J, et al. The regulation of the follicular synchronization and sensitivity of rats with PCOS by AMH during prolonged pituitary downregulation. Gene. $2019 ; 721: 144106$.

33. Dong M, et al. Regulation of AMH and SCF expression in human granulosa cells by GnRH agonist and antagonist. Pharmazie. 2011; 66(6):436-439.

34. Hernandez ER. Embryo implantation and GnRH antagonists: embryo implantation: the Rubicon for GnRH antagonists. Hum Reprod. 2000; 15(6):12111216.

35. Ruan $\mathrm{HC}$, et al. Ovarian stimulation with $\mathrm{GnRH}$ agonist, but not $\mathrm{GnRH}$ antagonist, partially restores the expression of endometrial integrin beta3 and leukaemia-inhibitory factor and improves uterine receptivity in mice. Hum Reprod. 2006; 21(10):2521-2529.

36. Chen $\mathrm{Q}$, et al. Comparative proteomics reveal negative effects of gonadotropin-releasing hormone agonist and antagonist on human endometrium. Drug Des Devel Ther. 2019; 13(1):1855-1863.

37. Huang J, et al. Comparison of the clinical application of GnRH antagonist protocol versus GnRH agonist long protocol in high responder PCOS patients. Guangdong Women and Children Hospital.

38. Ding W, et al. Impact of Female Obesity on Cumulative Live Birth Rates in the First Complete Ovarian Stimulation Cycle. Front Endocrinol (Lausanne). $2019 ; 10: 516$.

\section{Figures}




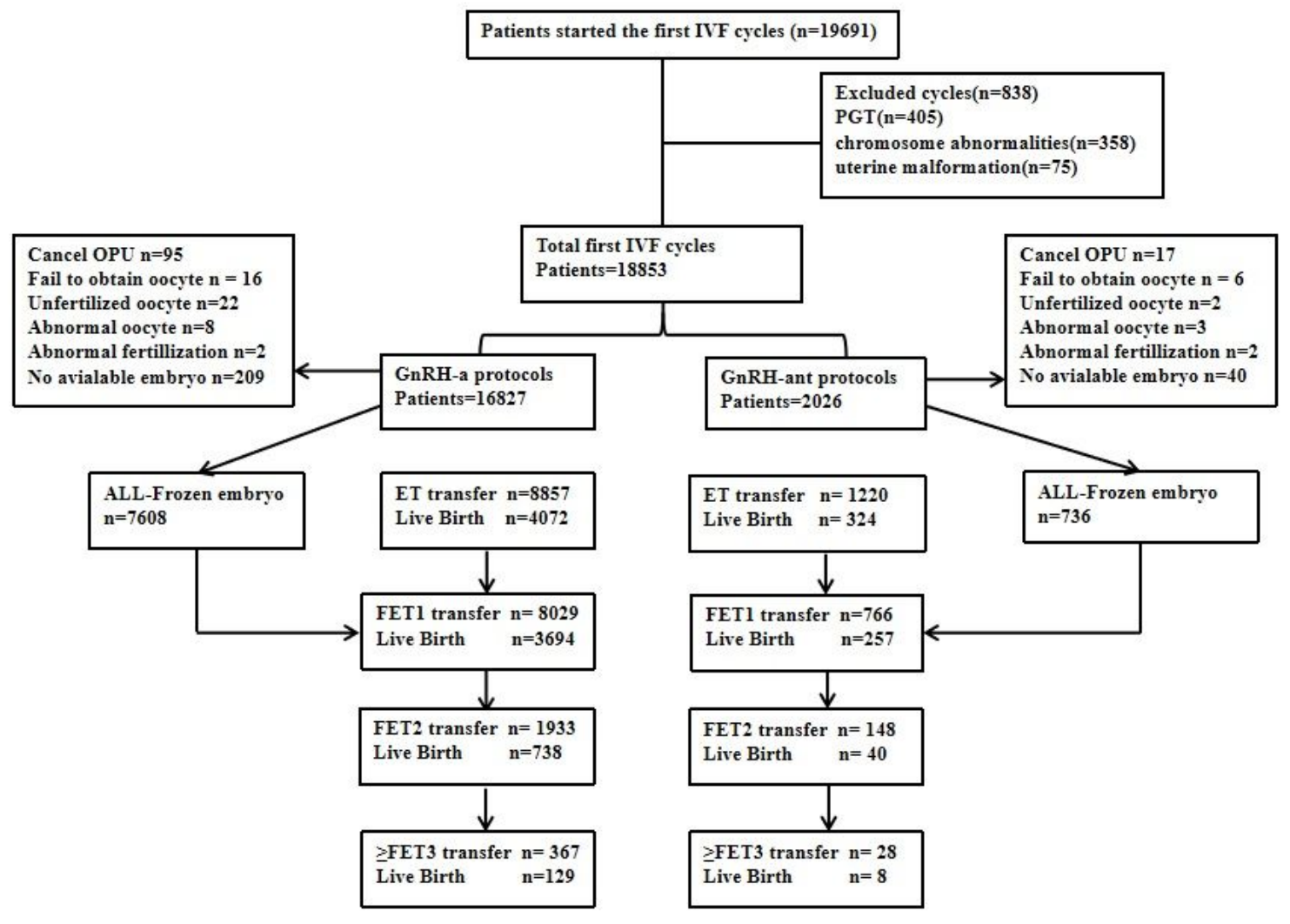

Figure 1

Flow chart

\section{Supplementary Files}

This is a list of supplementary files associated with this preprint. Click to download.

- SupplementalTable1.docx 